Suchen wir, Aerzte und Chemiker gemeinsam, die Frage in ihrer Einfachheit zu erhalten und energisch durchzuführen, so wird ein gater Fortschritt der Neuzeit erreicht werden.

Jena, im October 1872.

\title{
Receptblatt-Format und Recepten-Registratur.
}

Von Ludwig Leiner.

Zur vollständigen Ordnung in einem Apothekengeschäfte gehört so gut wie die in Einzelheiten durchgeführte Einrichtung der Officin, die Instandhaltung der verschiedenen Apparate zu pharmaceutischen, chemischen und mikroskopischen Arbeiten und ihre zeitweise Reform, auch die Ordnung in der Buchführung. Sie ist unumgehbar für die präcise Geschäfts Leitung. Und wie die Buchstaben - Ordnung zur - Wort - und Satz-Bildung führt, so ist auch die Ordnung der Recepte ein Theil der Basis der Buchfuihrang. Auch hier giebt es gewisse Gesetze, so einfach es scheint, so vornehm es oft vernachlässigt wird; und je ordentlicher es damit beschaffen ist, desto wohler ist es dem dabei, der Ordnung liebt.

Schon in dem 1856 herausgegebenen Versuche einer pharmaceutischen Buchführung*) ist auf zweckmässiges Format der Vormerkungsblätter hingewiesen und die ReceptenRegistratur kritischer behandelt, als es vordem Uebung war. Dort ist aber mehr ein allgemeiner Gesichtspunkt der Ueberschau und freien beliebigen Auswahl festgehalten worden. In der Besprechung einer Zeitschrift kann mehr der persönliche vertreten werden und kann jenen ergänzen.

1) Zur Ordnung der Registratur ist ein zweckmässiges Receptblatt-F ormat sehr wünschbar, und, mit Gründen

*) Versuch einer allgemeiner einzuführenden pharmaceutischen Buchfithrung von $B$ aur und $L$ einer. Leipzig $u$. Heidelberg; C. F. Winter'sche Verlagshandlung 1856. 
besprochen, dürfte es auch für allgemeinere Annahme Berücksichtigung finden. Unter Receptblättern verstehe ich hier sowohl die für ärztliche Ordinationen, deren Copieen, als auch die für Vormerkungen im Handverkauf bestimmten. Es ist mir in einer Recension damals entgegengehalten worden, dass diess ein der Buchung fremder Gegenstand sei. Und das ist es eben nicht. Jahrüber kommen aus Nord - und Süd-Deutschland und dem Auslande die sich für nachmalige Fascikulirung widerstreitendsten Formate von Receptblättern in die Apotheke; riemenförmige, auf denen der Arzt nicht einen einzigen Namen eines Arzneimittels, geschweige noch dessen verordnetes Gewicht, in eine Linie schreiben kann und das Papierbändchen schliesslich so vollgekritzelt ist, dass der Apotheker keinen Platz mehr für Taxation findet und für Repetitions - Vormerkungen besondere Blätter genommen werden müssen; oder man hat von Collegen in ihren Geschäften selbst eingeführte der verschiedensten oft unflätigsten Form vor sich. Da sind mit grossem Aufwande den dritten Theil des Zettels überdeckende Verzierungen, Engel, Mohren, Hirsche, Hof-Wappen, Basilisken und Drachen darauf lithographirt; und, für die Hauptsache wenig Platz gelassen, sind sie dadurch doch so gross, dass sie beim Einreihen in die Fascikel doppelt und dreifach eingebückt oder zugeschnitten werden müssen. Eine richtige Grösse findet sich aber so natürlich in dem doppelt über's Kreuz gehälfteten Bogen des allgemein üblichen Actenformats, der gesetzlich eingeführten Stempelbögen (34 Centim. hoch und 21,5 breit), also dann im Viertel von der Höhe von 17 und der Breite von 10,75 oder 11 Centim., dass man sich wundern muss, dass gerade diese zweckmässige Grösse wenigstens vielorts in der Minderheit bleibt. Auf solchen Blättern hat der Arzt und Apotheker hinreichend Platz, Ordination, Taxation und Repetitionen deutlich für's Auge auseinander zu halten. Eine edle Einfachheit, ohne viel Schnirkel und Zierath ist hiebei immerhin das Stilvollste und Schönste. Wer zieren will, wähle höchstens einen feinen zinnoberrothen Umfassungsstrich und in einfacher Schrift die Firma des Geschäftes. Die so 
224 Ludw. Leiner, Receptblatt-Format und Recepten-Registratur.

üblichen sonnenscheuen und den gemeinen Geschmack reizenden Anilin-Farben haben ihre Mode - Periode schon überlebt.

2) Da nun aber nicht Alle gleiche Ansicht haben, Viele oft recht Absonderliches lieben oder alter Gewohnheit fröhnen, so bleibt dem Apotheker, welcher es liebt, hübsche Recepten-Fascikel am Ende der Monate zu haben, nur übrig, zuzuschneiden und einzubïcken nach einem durchgreifenden Formate, für das ich eben das vorbezeichnete vorschlage. Dieses Zurichten der einzelnen auf Rechnung gebliebenen Vormerkblätter geschieht in meinem Geschäfte nach der Taxation täglich. Das Zusammenheften der Einzelblätter, die dem Originalformate nahegebracht sind, mache ich wieder ähnlich dem üblichen Fascikuliren der Acten, so dass alle Blätter auf der linken Lang- und obern Breit-Seite übereinander zu liegen kommen, gleich ob sie rechts und unten sich decken, und durchsteche sie in der linken oberu Ecke. Das Zusammenbinden geschieht mit einem dünnen mit Wachs gesteiften Faden, der geknüpft und nahe am Knopfe kurz abegeschnitten wird. Kommen im folgenden Monate neue Vormerkblätter dazu, so wird der Faden durchschnitten, mittelst Pfrieme und neuem Faden ebenso neu geheftet. Dickerer Bindfaden oder aufgeheftete Kartenblättchen, um die der Faden geschlungen wird, wie das Binden mit Schleifen trägt einseitig zu viel auf und ist beim Gebrauche hinderlich. Ebenso unpractisch halte ich das vielorts übliche Heften oben in der Mitte.

Jeder Fascikel erhält ein Deckblatt, welches in der obern Ecke rechts die Jahrszahl, in der Mitte den Namen des Kunden, und unten Bemerkungen über Wohnungswechsel, Zahlung oder Theilzahlung $u$. drgl. trägt in Geschäften, in denen kein solches verzeichnendes Conto-Corrent geführt wird. $\mathrm{Zu}$ Deutlichkeit und Schmuck trägt es viel bei, wenn hierbei die Vornamen mit dünnerer englischer, die Geschlechtsnamen mit der fettern rasch schreibbaren Rund-Schrift geschrieben werden.

3) Das Einreihender Kunden-Fascikel im Buchstaben-Fascikel geschieht streng-alphabetisch und diese 
Ordnung soll fest eingehalten werden. Nur so ist es möglich auf Jahre zurïck immer auf die ersten Auseinanderschlagungen sofort die gesuchten Recepten-Bündel zur Hand zu haben. Die Buchstaben-Fascikel lege ich so geordnet je zwischen zwei lose Pappendeckel, deren oberer den Buchstaben und die Jahreszahl trägt. Ein einfacher starker Kautschuk-Ring hält den Fascikel zusammen. So liegen die Bündel gleichförmig, festzusammengehalten und immer ordentlich in den Schiebladen der Registratur.

4) Das Notiren zu Buch ist Eigensache der im einzelnen Geschäfte eingeführten Ordnung.

So einfach und naheliegend solche Recepten-Anordnung ist, so habe ich bezüglich Format und Registratur die vorschiedensten mir weniger zusagenden Arten gefunden und halte eine Besprechung der Sache als Anregung zu Meinungsaustausch in den Spalten einer Apothekerzeitschrift am Platz.

\section{Ausbeuten an Extract.}

Aus dem pharmaceutischen Laboratorium.

Von Hermann Werner, Apotheker in Breslau.

Durch den Umstand, unter anderen pharmaceutischen Präparaten auch Extracte an hiesige Droguenhäuser zu liefern, bin ich in der Lage, dieselben in grösseren Quantitäten und öfters darzustellen. Nicht in der Absicht, die Resultate meiner Arbeiten zu veröffentlichen, sondern um bei wiederholter Bereitung die gewonnenen Ausbeuten vergleichen zu können, machte ich mir über den Verlauf und das Endresultat der Arbeiten genauere Notizen. Die in letzterer Zeit in unseren Fachjournalen mehrfach gefundenen Notizen über Ausbeute an Extracten veranlassten mich, meine Arbeiten mit diesen und mit denen in Hag er's Manuale pharmaceuticum II zu vergleichen; und wenn ich dem auf diesem Gebiet reichen Arch, . Pharm. IIT. Relho, II, Bis, 3 Hrt. 